\title{
Adverse Events Following COVISHIELD Vaccination Among Adult Population in Bangladesh
}

\author{
Md. Musab Khalil ${ }^{1}$ (D) $\cdot$ Khandker Mahbub-Uz-Zaman $^{1}$ (D) $\cdot$ As-Saba Hossain ${ }^{2} \cdot$ Farid Ahmed $^{1}$. \\ Md. Fazlul Karim Chowdhury ${ }^{1}$ (D) Sharmin Tahmina Khan ${ }^{1} \cdot$ Md. Shah Alam Miah ${ }^{1} \cdot$ Narwana Khaleque $^{1}$. \\ Md. Golam Kibria ${ }^{1}$. Faruque Ahmed ${ }^{1} \cdot$ Ahad Mahmud Khan $^{3}$ (I)
}

Accepted: 7 July 2021 / Published online: 31 July 2021

(C) The Author(s) 2021

\begin{abstract}
The study aimed to determine how frequently the adverse events of the COVISHIELD vaccine occur among the Bangladeshi population. This cross-sectional study was conducted at Sheikh Russel Gastroliver Institute and Hospital, Mohakhali, Dhaka, Bangladesh, in May 2021. The inclusion criteria were the adult populations who received the 2nd dose of the COVISHELD vaccine and had passed 28 days following the completion of the 2 nd dose. Three hundred and five persons fulfilling the inclusion criteria were asked over the telephone - based on a predesigned questionnaire. The rates of adverse events were $54.1 \%$ and $41.3 \%$ after the 1 st and 2 nd dose of vaccine, respectively, and the difference was statistically significant $(p<0.001)$. Pain at the injection site was the most common adverse event (32.5\% following the 1st dose and 27.9\% following the 2 nd dose). All of the symptoms were mild and lasted for about 2 days. Age and comorbidities were significantly associated with the adverse events $(p$ $<0.001)$. Neither doses had any vaccine-related life-threatening adverse event nor had any symptoms related to vaccine-related blood clotting. Nineteen persons (6.2\%) had been diagnosed with COVID-19 after the 1st dose of vaccination, and three (1\%) persons had been diagnosed with COVID-19 after the 2nd dose of vaccination. As no significant life-threatening adverse event was observed, this study might help reduce the hesitancy for vaccination among the population and thus help reduce transmission of this highly contagious virus.
\end{abstract}

Keywords COVID $19 \cdot$ Vaccination · Adverse Events · COVISHIELD · Oxford AstraZeneca

\section{Introduction}

Coronavirus disease 2019 (COVID-19) has significantly damaged the world population with mortality and morbidity of unprecedented scale. As of 03 May 2021, more than 150 million people have been confirmed to be identified as being infected with the disease, and about 3.2 million people succumbed to death because of this disease [1]. COVID-19 is caused by SARS-COV-2, an enveloped, positive-sense

This article is part of the Topical Collection on COVID-19

Ahad Mahmud Khan

ahad_mahmud@hotmail.com; A.M.Khan@sms.ed.ac.uk

1 Sheikh Russel Gastroliver Institute and Hospital, Dhaka, Bangladesh

2 American International University of Bangladesh,

Dhaka, Bangladesh

3 The University of Edinburgh, Edinburgh, UK single-stranded RNA virus, which has a glycoprotein structure resembling spikes on the surface, responsible for the attachment with receptor and cellular invasion of the host $[2,3]$. Patients diagnosed with COVID-19 usually suffer from fever, cough, and shortness of breath, along with other symptoms. Although predominantly a respiratory tract infection, it affects other organs as well. The death of the patients was attributed to the development of multiple organ failure. Dysregulated immune-thrombosis involving neutrophils and platelets has been designated as the reason for increased incidents of acute respiratory distress syndrome (ARDS) and systemic hypercoagulability [4-6].

Currently, there is no specific treatment for this deadly disease. Implementation of different prevention modalities has been put in place to interrupt the transmission of the disease. Vaccination is a key feature among these modalities. As of 04 May 2021, 13 different vaccines are being used globally. Of these, two are mRNA vaccines, four are vector (nonreplicating) vaccines, five are inactivated, and two are protein subunit vaccines. Over one billion dosages of vaccines have 
been administered [7]. It has been shown that frontline workers are at risk of contracting the infection because of the limited supply of personal protective equipment (PPE) [8]. Hence, for the safeguard of the healthcare workers and to ensure the interruption of the transmission cycle, vaccination holds the best approach for the prevention and control of the disease [9].

Bangladesh introduced vaccination for the vulnerable groups, frontline workers, and emergency personnel on 27 January 2021 and nationwide vaccination drive from 07 February 2021 with the vaccine COVISHEID based on ChAdOx1 $\mathrm{nCoV}-19$ vaccine that has been developed by The Jenner Institute, Oxford University, with the technical support from AstraZeneca and being produced by Serum Institute of India, Pune, Maharashtra, India. Bangladesh purchased this vaccine from the Serum Institute of India [10].

This ChAdOx $1 \mathrm{nCoV}-19$ vaccine consists of the replicationdeficient simian adenovirus vector ChAdOx1, containing the full-length structural surface glycoprotein (spike protein) of SARS-CoV-2, with a tissue plasminogen activator leader sequence. ChAdOx1 nCoV-19 expresses a codon-optimized coding sequence for the spike protein [11]. Bangladesh is to receive a total number of 68 million doses of vaccine as a target of vaccinating the $30 \%$ vulnerable population as well as protecting the frontline workers [12]. As this is an unprecedented event, it is important to follow up the safety of the vaccine for all the adverse events to be recorded and stored, and also keep track of the severe adverse events following the vaccination as part of vaccine surveillance so that any damage could be minimized and adequate steps could be taken beforehand.

Adult vaccination drive has never been undertaken on such a scale. Therefore, the acceptability of the vaccine as well as the safety concern regarding the vaccine has to be evaluated. Even though there has been some acceptability, vaccine hesitancy, defined as the vaccine acceptance is prolonged or refusal to accept the service of vaccination, is a feature among the people [13]. In a cross-sectional study, it has been shown to have $61.34 \%$ acceptability of the vaccine, but only $35.14 \%$ are willing to accept it [14].

There is also a need to rule out certain misconceptions regarding the side effects of the vaccine that need to be addressed to prevent vaccine hesitancy. Therefore, this study was conducted among vaccinated persons regarding the post-vaccination adverse events and severe adverse events for the safety of the vaccinated persons as well as for future policy planning and implementation.

\section{Methods}

\section{Study Design, Setting, and Participants}

This cross-sectional study was conducted at Sheikh Russel Gastroliver Institute and Hospital (SRGIH), Mohakhali,
Dhaka, Bangladesh, from 8 to 15 May 2021. A total of 350 persons aged 18 years and older received the 2nd dose of COVISHIELD vaccine, and in 28 days passed from the last dose, they were approached over the telephone.

\section{Sampling Technique}

A list of persons who completed the 2nd dose of vaccine and completed 28 days post-vaccination was obtained from the hospital records. A consecutive sampling technique was applied. These 350 vaccine recipients were approached over the phone to collect data from them. At least three attempts of calling were made at least $1 \mathrm{~h}$ apart before telling non-response. Forty-five persons did not respond to the call. Finally, 305 persons were enrolled.

\section{Data Collection}

Data collection was done between the 30th and 35th day following 2 nd dose of vaccination. The participants were asked over the telephone using a predesigned structured questionnaire. Data regarding sociodemographic characteristics, comorbidities, adverse events of vaccination (both the doses), and diagnosis of COVID-19 were collected. The types of adverse events collected were local, general, medically attended, and serious adverse events. The local adverse events included pain, swelling, itching, and rash at the injection site. General adverse events included fever, chills and rigors, generalized body ache, fatigue, malaise, somnolence, drowsiness, insomnia, headache nausea, vomiting, diarrhea, dizziness, joint pain, runny nose, and redness of the eyes. Medically attended adverse event was defined if a medical practitioner was consulted for managing the adverse events. An event was considered serious if it resulted in death, was life-threatening, required in-patient hospitalization or prolongation of existing hospitalization, resulted in persistent or significant disability/ incapacity, or was a congenital anomaly/birth defect [15]. The severity of the adverse events was assessed by numerical rating scale (NRS) starting from 0 to 10 [16]. Zero was depicted as having no symptom, 1-3 as mild symptoms, 4-6 as having moderated symptoms, and $7-10$ as severe symptoms [17]. The diagnosis of COVID-19 was based on RT PCR for SARSCOV-2 test [18].

\section{Statistical Analysis}

Collected data were cleaned and assessed for distribution. All quantitative data were non-normally distributed except the age of the study participants. Quantitative data were expressed in mean $\pm \mathrm{SD}$, and qualitative data were expressed in percentage. Wilcoxon paired signed ranks test was used to compare paired data. Mann-Whitney $U$ test was used to compare two groups of quantitative variables. Binary logistic regression was done 
to assess the predictor of adverse events of vaccination. A two-tailed value of $p \leq 0.05$ was considered statistically significant for all analyses.

\section{Results}

Among the 305 participants, 173 were males (56.4\%), with their mean age being 47 years (SD 13.6) (Table 1). The rates of adverse events were $54.1 \%$ and $41.3 \%$ after the 1 st and 2 nd doses of vaccine, respectively. The adverse events were significantly higher following the 1 st dose compared to the 2 nd dose $(p<0.001)$. The most frequent adverse event was pain at the injection site that was reported among 99 (32.5\%) after the 1 st dose and 85 (27.9\%) after the 2 nd dose. Fever was recorded among $61(20 \%)$ in 1st dose and $37(12.1 \%)$ in the 2nd dose. Fatigue, malaise, generalized body ache, and headache were recorded $6.9 \%, 16.4 \%, 16.4 \%$, and $6.9 \%$, respectively, following the 1 st dose of vaccination, while after the 2 nd dose, these were reported $3.3 \%, 8.2 \%, 9.5 \%$, and $5.2 \%$ which showed the adverse events occurred in significantly less during the second vaccination $(p<0.001)$. However, none of the participants had severe life-threatening adverse events. None of them had symptoms related to thrombosis as well. The symptoms were mild based on the NRS that lasted mostly 2 days. Furthermore, almost one-fifth of the participants needed medications, namely paracetamol, to get rid of the symptoms. Few participants had to consult with the medical practitioners (8.2\% after the 1 st dose and $3.9 \%$ following the second dose). Significant numbers of the participants had their daily activities hampered following the 1st dose in contrast to the second dose $(p<0.007$ vs. $p<0.832)$. Additionally, the severity and duration of adverse events and adverse events hampering daily activities were more with the 1 st dose, and the difference was statistically significant (Table 2).

Moreover, age and comorbidity had been significantly associated with the vaccine-related adverse events (Table 3). Almost half of the participants had comorbidities. Among the comorbid populations, hypertension was the most prevalent (35.4\%), followed by diabetes mellitus, which constituted one-fourth of the comorbid population (23.3\%). More than two-thirds of them were non-smokers. Following the adverse events, the mean duration of the symptoms persisted $1.9 \pm 1.3$ days $(p<0.01)$ for the 1 st dose of vaccination while $1.7 \pm 0.9$ days $(p<0.01)$ following the 2 nd dose of vaccination.

Nineteen (6.2\%) had COVID-19 after the 1st dose, and 3 (1\%) participants had COVID-19 after the 2nd dose (Figure 1). Among the participants diagnosed with COVID19 following vaccination, the majority had been diagnosed with COVID-19 after the second week. None of the patients who suffered from COVID-19 became critically ill (Table 4).

\section{Discussion}

This study demonstrates that the rates of adverse events of the COVISHIELD vaccine were $54.1 \%$ after the 1 st dose and $41.3 \%$ after the 2 nd dose of the vaccine. However, these were minor adverse events like pain at the injection site, fever, feeling unwell, and generalized body ache. There were no
Table 1 Sociodemographic factors and comorbidities $(n=$ 305)

\begin{tabular}{lll}
\hline Age, mean \pm SD (in years) & & $47.3 \pm 13.6$ \\
\hline Gender, $n(\%)$ & Male & $173(56.7)$ \\
Occupation, $n(\%)$ & Female & $132(43.3)$ \\
& Doctor & $35(11.5)$ \\
& Nurse & $19(6.2)$ \\
& Pharmacist & $6(1.9)$ \\
& Healthcare professional (other than doctor, nurse, and pharmacist) & $19(6.2)$ \\
& Service & $80(26.2)$ \\
& Business & $43(14.1)$ \\
& Housewife & $75(24.6)$ \\
& Retired from service & $11(3.6)$ \\
& Others* & $17(5.6)$ \\
Smoking habit, $n(\%)$ & Smoker & $40(13.1)$ \\
& Ex-smoker & $21(6.9)$ \\
& Non-smoker & $244(80)$ \\
Comorbidity, $n(\%)$ & Present & $152(49.8)$ \\
& Absent & $153(50.2)$ \\
\hline
\end{tabular}

*Engineer, chartered accountant, architect, school teacher, banker, driver 
Table 2 Adverse events after vaccination $(n=305)$

\begin{tabular}{|c|c|c|c|}
\hline & After 1st dose & After 2nd dose & $p^{*}$ \\
\hline Adverse events occurred, $n(\%)$ & $165(54.1)$ & $126(41.3)$ & $<0.001$ \\
\hline Pain at injection site, $n(\%)$ & $99(32.5)$ & $85(27.9)$ & \\
\hline Fever, $n(\%)$ & $61(20)$ & $37(12.1)$ & \\
\hline Fatigue, $n(\%)$ & $21(6.9)$ & $10(3.3)$ & \\
\hline Malaise, $n(\%)$ & $50(16.4)$ & $25(8.2)$ & \\
\hline Generalized body ache, $n(\%)$ & $50(16.4)$ & $29(9.5)$ & \\
\hline Headache, $n(\%)$ & $21(6.9)$ & $16(5.2)$ & \\
\hline Chills and rigor, $n(\%)$ & $9(3)$ & $5(1.6)$ & \\
\hline Swelling at injection site, $n(\%)$ & $9(3)$ & $2(0.7)$ & \\
\hline Itching and rash at injection site, $n(\%)$ & $0(0.0)$ & $1(0.3)$ & \\
\hline Dizziness, $n(\%)$ & $3(1)$ & $4(1.3)$ & \\
\hline Nausea, n (\%) & $8(2.6)$ & $5(1.6)$ & \\
\hline Vomiting, $n(\%)$ & $2(0.7)$ & $2(0.7)$ & \\
\hline Diarrhea, $n(\%)$ & $2(0.7)$ & $1(0.3)$ & \\
\hline Somnolence, $n(\%)$ & $2(0.7)$ & $1(0.3)$ & \\
\hline Drowsiness, $n(\%)$ & $1(0.3)$ & $0(0.0)$ & \\
\hline Runny nose, $n(\%)$ & $2(0.7)$ & $0(0.0)$ & \\
\hline Redness of the eye, $n(\%)$ & $1(0.3)$ & $0(0.0)$ & \\
\hline Joint pain, $n(\%)$ & $3(1)$ & $1(0.3)$ & \\
\hline Insomnia, $n(\%)$ & $0(0.0)$ & $1(0.3)$ & \\
\hline Severity of adverse event in NRS, mean \pm SD & $2.6 \pm 1.5$ & $2.5 \pm 1.6$ & 0.027 \\
\hline Duration of adverse event, mean $\pm \mathrm{SD}$ & $1.9 \pm 1.3$ & $1.7 \pm 0.9$ & 0.01 \\
\hline Medication needed to manage adverse event, $n(\%)$ & $79(25.9)$ & $60(19.7)$ & 0.003 \\
\hline $\begin{array}{l}\text { Consulted medical practitioner for managing } \\
\quad \text { adverse event, } n(\%)\end{array}$ & $25(8.2)$ & $12(3.9)$ & 0.009 \\
\hline Adverse event hampered daily activity, $n(\%)$ & $70(23 \%)$ & $31(10.2)$ & $<0.001$ \\
\hline \multirow[t]{2}{*}{ Adverse event hampered daily for how many days, mean \pm SD } & $1.6 \pm 1.2$ & $1.5 \pm 1.2$ & 0.083 \\
\hline & $\begin{array}{l}\text { Severity of adverse event in NRS } \\
\text { Mean } \pm \text { SD } p^{* *}\end{array}$ & $\begin{array}{l}\text { Severity of adverse event in NRS } \\
\text { Mean } \pm \mathrm{SD} \quad p^{* *}\end{array}$ & \\
\hline \multirow[t]{2}{*}{ Adverse event hampered daily activity } & $3.1 \pm 1.8(n=60)$ & $2.7 \pm 1.9(n=31)$ & \\
\hline & $2.3 \pm 1.2(n=105)$ & $2.5 \pm 1.4(n=95)$ & \\
\hline
\end{tabular}

*Wilcoxon paired signed ranks test, **Mann-Whitney $U$ test, $p \leq 0.05$ considered significant. $N R S$, numerical rating scale

Table 3 Factors predicting adverse events of vaccine $(n=305)$

\begin{tabular}{|c|c|c|c|c|c|c|c|c|c|}
\hline \multirow[b]{2}{*}{ Factor } & \multicolumn{5}{|c|}{1 st dose of vaccine, model $x^{2}(16.8, p 0.005)$} & \multicolumn{4}{|c|}{ 2nd dose of vaccine, model $x^{2}(32.8, p<0.001)$} \\
\hline & Variable coding for factor & $B$ & S.E. & OR & ${ }_{\mathrm{P}} \mu$ & $B$ & S.E. & OR & ${ }_{\mathrm{P}} \mu$ \\
\hline Age & & 0.036 & 0.011 & 1.037 & 0.001 & 0.060 & 0.012 & 1.062 & $<0.001$ \\
\hline Gender & $\begin{array}{l}\text { Male }=1 \\
\text { Female }=2\end{array}$ & -0.432 & 0.266 & 0.649 & 0.104 & 0.089 & 0.278 & 1.093 & 0.748 \\
\hline Non-smoker & & Constant & & & 0.278 & & & & 0.806 \\
\hline Ex-smoker & & -0.479 & 0.382 & 0.619 & 0.210 & -0.075 & 0.393 & 0.928 & 0.849 \\
\hline Smoker & & -0.603 & 0.496 & 0.547 & 0.225 & -0.338 & 0.517 & 0.713 & 0.514 \\
\hline Comorbidity & $\begin{array}{l}\text { Present }=1 \\
\text { Absent }=2\end{array}$ & 0.594 & 0.278 & 1.811 & 0.033 & 1.226 & 0.303 & 3.408 & $<0.001$ \\
\hline
\end{tabular}

$\mu$, binary logistic regression; $p \leq 0.05$, considered significant; $B$, regression coefficient; $O R$, odds ratio; S.E.-Standard error 


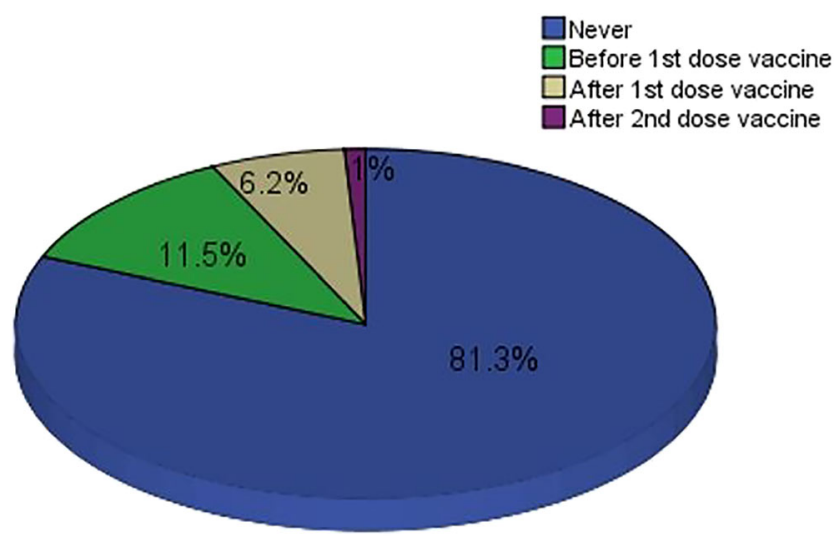

Fig. 1 Time of diagnosis of COVID-19 in relation to vaccination, $n=305$

significant life-threatening adverse events after vaccine administration.

Given the outcome of the phase 3 trial in the United Kingdom (UK), Brazil, and South Africa, the ChadOx1 vaccine had demonstrated $63 \%$ efficacy against symptomatic COVID-19 cases, with acceptable tolerability and no serious adverse event related to vaccination [19,20]. Adverse events recorded in our study were compared to the anticipated adverse events by the World Health Organization (WHO) SAGE working group based on phase 3 clinical trials in the UK, Brazil, and South Africa and phase $1 / 2$ trial in the UK, and there were significant differences [19-21]. In the WHO Working Group, the headache had been observed among $52.6 \%$ of participants, while the phase $1 / 2$ trial found $68 \%$ of participants $[19,20]$.
Compared to that, our study found only $6.9 \%$ of the study population complained of headache following the vaccination. Another adverse event chill was present among $31.9 \%$ by the WHO Working Group paper and 56.4\% among the phase 1/2 trial, while our study population only reported $3 \%$ following vaccination. Pain at the injection site was present among $32.5 \%$ of participants from our study, while compared that with the WHO Working SAGE Working Group paper and phase $1 / 2$ trial in the UK reported $54.2 \%$ and $67 \%$, respectively. Nausea as an adverse event following the vaccination had been reported only among $2.6 \%$ of our participants; compared that with the WHO SAGE Working Group and phase 1/2 trial in the UK, it was reported $21.9 \%$ and $25 \%$, respectively [19-21]. Phase $1 / 2$ trial in the UK found generalized body pain among $60 \%$ of participants, and the WHO SAGE Working Group paper anticipated this adverse event to be reported among $44 \%$ of the population. In our study, we only recorded $16.4 \%$ population reported this adverse event [19-22]. Figure 2 shows the comparison of adverse events reported in our study with the study of the WHO SAGE Working Group [19-21] and Folegatti et al. [22].

When comparing the adverse event from the 1st dose and 2nd dose of vaccination, according to the WHO SAGE Working Group, adverse events that occurred more than $10 \%$ are being denoted as very common, and from 1 to $10 \%$ are being termed as common. By that classification, pain at the injection site (32.5\%), fever (20\%), malaise (16.4\%), and generalized body ache $(16.4 \%)$ were the very common adverse events following the 1 st dose of vaccination, while fatigue $(6.9 \%)$, headache $(6.9 \%)$, chill (3\%), swelling at the injection site $(3 \%)$, dizziness $(1 \%)$, and nausea $(2.6 \%)$ were common

Table 4 Diagnosis of COVID-19 after the 1 st or 2 nd dose of the vaccine $(n=22)$

\begin{tabular}{llll}
\hline & & $\begin{array}{l}\text { After 1st dose of vaccine } \\
(n=19)\end{array}$ & $\begin{array}{l}\text { After 2nd dose of vaccine } \\
(n=3)\end{array}$ \\
\hline Vaccination to symptom onset of COVID-19, $n(\%)$ & Asymptomatic & $1(5.3)$ & $0(0.0)$ \\
& Within 2 weeks of the vaccine dose & $3(15.8)$ & $1(33.3)$ \\
& After 2 weeks of the vaccine dose & $15(78.9)$ & $2(66.7)$ \\
Duration of symptoms of COVID-19, $n(\%)$ & No symptom & $2(10.5)$ & $1(33.3)$ \\
& $\leq 7$ days & $13(68.4)$ & $1(33.3)$ \\
& $8-14$ days & $4(1.3)$ & $1(33.3)$ \\
Treated at, $n(\%)$ & $>14$ days & $0(0.0)$ & $2(66.7)$ \\
Needed oxygen, $n(\%)$ & Home & $15(4.9)$ & $1(33.3)$ \\
Needed HDU/ICU, $n(\%)$ & Yespital & $4(21.1)$ & $1(33.3)$ \\
Needed intubation, $n(\%)$ & No & $3(15.8)$ & $2(66.7)$ \\
& Yes & $16(84.2)$ & $0(0.0)$ \\
& No & $0(0.0)$ & $3(100)$ \\
\end{tabular}


Figure 2 Comparison of adverse events reported in the study with WHO SAGE Working Group AZD-1222 vaccine against COVID-19 and Folegatti et al. [22]

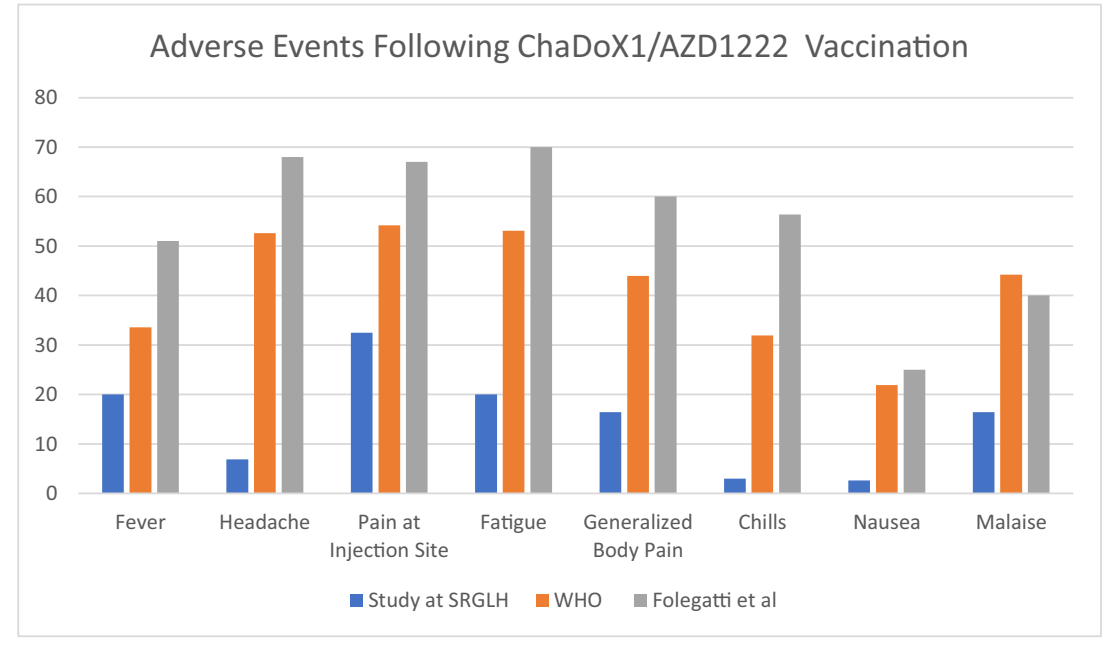

adverse events in our study. Following the 2nd dose of vaccination, only pain at the injection site $(27.9 \%)$ and fever $(12.1 \%)$ are denoted as very common adverse events, and the rest can be described as common adverse events [21, 20].

Our study data following binary logistic regression suggested comorbidity had a significant relation with the adverse event following the 1 st and 2 nd dose of vaccination with odds ratio for the 1 st dose being 1.8 with S.E. $0.278, p<0.005$, and for the second dose, odds ratio is 3.408 , S.E. 0.303 , and $p<$ 0.001 . Our finding for the duration symptoms persisted more than 7 days for only 4 participants following the 1 st dose of vaccination while only 2 participants reported symptoms more than 7 days following the 2 nd dose vaccination, which was lower than what the WHO SAGE Working Group showed with the persistence of $13 \%$ following day 7 [19]. After the 1 st dose of vaccination, the mean duration of adverse events following vaccination is $1.9 \pm 1.3$ days, while after the 2 nd dose of vaccination, the mean duration is $1.6 \pm .09$ days. The adverse events severity as well persistence had been less in our study population compared to the WHO SAGE Working Group paper based on multiple trials conducted in the UK, South Africa, and Brazil [19, 22, 20].

For the adverse events, only 25 participants consulted the physicians for their symptoms where 79 persons reported to have taken medication for the relief of symptoms and 70 participants reported hampered daily activity, with mean of $1.6 \pm$ 1.3 days following the first dose, compared to $1.5 \pm 1.2$ days following the 2 nd dose. After the 2 nd dose, the report of hampering of daily activity had been reported by 31 participants. Like the adverse events which were reported significantly less than the 1st dose, the severity and hampering daily activity showed the same trend. The severity of the adverse event hampering daily activity following 1 st dose vaccination had been shown statistically significant mean of $3.1 \pm 1.8$ days $(p<0.007)$, which was not the case following the 2 nd dose. It also solidifies the fact that the severity of the adverse events was significantly less than in the 1st dose of vaccination [20].

\section{Limitation of the Study}

No immediate allergic reaction following vaccination was recorded, and there had not been any incidents of anaphylactic reaction or shock following the vaccination. This also denotes the allergic reaction to the components of the vaccine is minimum, but the scale of the population may give us limited knowledge. During the initial phase of the vaccine development, it has been privy to transverse myelitis and pyrexia, which has not been demonstrated among our study population. Immunothrombosis following vaccination had been reported as a very rare adverse event following vaccination among the population with low platelet count; however, none of the members of the study population had shown any sign of such condition. As these are very rare adverse events, our study population data may be limited in this regard and may require further study with a larger population.

\section{Conclusion}

These observations exemplify the fact that the COVISHIELD vaccine is a well-tolerated and safe vaccine, which can be administered among the adult population of Bangladesh. As no significant life-threatening adverse event was observed, this study might help reduce hesitancy for vaccination among the population and thus help reduce transmission of this highly contagious disease.

Acknowledgements The authors would like to acknowledge all respected respondents for their valuable time and participation in this research work.

Author Contribution MMK, ASH, AMK, FA, and MGK formed the idea of this study. MMK, FAT, FKC, STK, SAM, NKM, and KMZ collected the data. $\mathrm{KMZ}$ and ASH performed the analysis. ASH, MMK, KMZ, and 
AMK prepared the manuscript. All the authors reviewed and approved the final manuscript.

Data Availability These are available upon reasonable request.

Code Availability Not applicable

\section{Declarations}

Ethics Approval Ethical approval was obtained from the ethical review committee of Sheikh Russel National Gastroliver Institute and Hospital, Mohakhali, Dhaka.

Consent to Participate Informed consent was taken from each participant.

Consent for Publication The authors have full right to publish the manuscript.

Competing Interests The authors declare no competing interests.

Open Access This article is licensed under a Creative Commons Attribution 4.0 International License, which permits use, sharing, adaptation, distribution and reproduction in any medium or format, as long as you give appropriate credit to the original author(s) and the source, provide a link to the Creative Commons licence, and indicate if changes were made. The images or other third party material in this article are included in the article's Creative Commons licence, unless indicated otherwise in a credit line to the material. If material is not included in the article's Creative Commons licence and your intended use is not permitted by statutory regulation or exceeds the permitted use, you will need to obtain permission directly from the copyright holder. To view a copy of this licence, visit http://creativecommons.org/licenses/by/4.0/.

\section{References}

1. Worldometer. https://www.worldometers.info/coronavirus/.

2. Gao Y, Yan L, Huang Y, Liu F, Zhao Y, Cao L, et al. Structure of the RNA-dependent RNA polymerase from COVID-19 virus. Science. 2020;368(6492):779-82.

3. Yuki K, Fujiogi M, Koutsogiannaki S (2020) COVID-19 pathophysiology: a review. Clin Immunol:108427

4. Chen N, Zhou M, Dong X, Qu J, Gong F, Han Y, et al. Epidemiological and clinical characteristics of 99 cases of 2019 novel coronavirus pneumonia in Wuhan, China: a descriptive study. Lancet. 2020;395(10223):507-13.

5. Khalil M, Gain G, Mahbub-Uz-Zaman K, Karim M, Sarker K, Akter D, et al. Gastrointestinal manifestations among COVID-19 patients in Bangladesh: a cross sectional study. Mymensingh Med J. 2020;29(4):956-63.

6. Nicolai L, Leunig A, Brambs S, Kaiser R, Weinberger T, Weigand $\mathrm{M}$, et al. Immunothrombotic dysregulation in COVID-19 pneumonia is associated with respiratory failure and coagulopathy. Circulation. 2020;142(12):1176-89.
7. COVID-19 vaccine tracker. https://vac-lshtm.shinyapps.io/ncov_ vaccine landscape/.

8. Khalil MM, Alam MM, Arefin MK, Chowdhury MR, Huq MR, Chowdhury JA, et al. Role of personal protective measures in prevention of COVID-19 spread among physicians in Bangladesh: a multicenter cross-sectional comparative study. SN Comprehen Clin Med. 2020;2(10):1733-9.

9. Shekhar R, Sheikh AB, Upadhyay S, Singh M, Kottewar S, Mir H, et al. COVID-19 vaccine acceptance among health care workers in the United States. Vaccines. 2021;9(2):119.

10. Sattar AA. Prons and cons of Covid19 vaccination: Bangladesh perspectives. J Curr Adv Med Res. 2021;8(1):1-2.

11. Watanabe Y, Mendonça L, Allen ER, Howe A, Lee M, Allen JD, et al. Native-like SARS-CoV-2 spike glycoprotein expressed by ChAdOx1 nCoV-19/AZD1222 vaccine. ACS Central Sci. 2021;7(4):594-602.

12. COVAX explained. https://www.gavi.org/vaccineswork/covaxexplained.

13. Mahmud S, Mohsin M, Khan IA, Mian AU, Zaman MA (2021) Acceptance of COVID-19 vaccine and its determinants in Bangladesh. arXiv preprint arXiv:210315206

14. Riad A, Pokorná A, Attia S, Klugarová J, Koščík M, Klugar M. Prevalence of COVID-19 vaccine side effects among healthcare workers in the Czech Republic. J Clin Med. 2021;10(7):1428.

15. World Health Organization (2020) COVID-19 vaccines: safety surveillance manual.

16. Hjermstad MJ, Fayers PM, Haugen DF, Caraceni A, Hanks GW, Loge JH, et al. Studies comparing Numerical Rating Scales, Verbal Rating Scales, and Visual Analogue Scales for assessment of pain intensity in adults: a systematic literature review. J Pain Symptom Manag. 2011;41(6):1073-93.

17. Pantilat SZ, O'Riordan DL, Dibble SL, Landefeld CS. Longitudinal assessment of symptom severity among hospitalized elders diagnosed with cancer, heart failure, and chronic obstructive pulmonary disease. J Hosp Med. 2012;7(7):567-72.

18. Poortahmasebi V, Zandi M, Soltani S, Jazayeri SM. Clinical performance of RT-PCR and chest CT scan for COVID-19 diagnosis; a systematic review. Adv J Emerg Med. 2020;4(2s):e57.

19. Ramasamy MN, Minassian AM, Ewer KJ, Flaxman AL, Folegatti PM, Owens DR, et al. Safety and immunogenicity of ChAdOx1 $\mathrm{nCoV}-19$ vaccine administered in a prime-boost regimen in young and old adults (COV002): a single-blind, randomised, controlled, phase 2/3 trial. Lancet. 2020;396(10267):1979-93.

20. World Health Organization (2021) COVID-19 vaccine explainer COVID-19 vaccine ChAdOx1-S [recombinant], Developed by Oxford University and AstraZeneca.

21. World Health Organization Background paper on COVID-19 vaccines prepared by the Strategic Advisory Group of Experts (SAGE) on Immunization Working Group on COVID-19 vaccines. https:// covid19.who.int/table.

22. Folegatti PM, Ewer KJ, Aley PK, Angus B, Becker S, BelijRammerstorfer S, et al. Safety and immunogenicity of the ChAdOx1 nCoV-19 vaccine against SARS-CoV-2: a preliminary report of a phase $1 / 2$, single-blind, randomised controlled trial. Lancet. 2020;396(10249):467-78.

Publisher's Note Springer Nature remains neutral with regard to jurisdictional claims in published maps and institutional affiliations. 